\title{
THE EFFECTS OF BINARY EVOLUTION ON THE DYNAMICS OF CORE COLLAPSE AND NEUTRON STAR KICKS
}

\author{
Ph. Podsiadlowski, ${ }^{1}$ N. Langer, ${ }^{2}$ A. J. T. Poelarends, ${ }^{2}$ S. Rappaport, ${ }^{3}$ A. Heger,${ }^{4}$ and E. Pfahl ${ }^{5}$ \\ Received 2003 September 22; accepted 2004 March 31
}

\begin{abstract}
We systematically examine how the presence in a binary affects the final core structure of a massive star and its consequences for the subsequent supernova explosion. Interactions with a companion star may change the final rate of rotation, the size of the helium core, the strength of carbon burning, and the final iron core mass. Stars with initial masses larger than $\sim 11 M_{\odot}$ that experience core collapse will generally have smaller iron cores at the point of explosion if they lost their envelopes through a binary interaction during or soon after core hydrogen burning. Stars below $\sim 11 M_{\odot}$, on the other hand, can end up with larger helium and metal cores if they have a close companion, since the second dredge-up phase that reduces the helium core mass dramatically in single stars does not occur once the hydrogen envelope is lost. We find that the initially more massive stars in binary systems with masses in the range 8-11 $M_{\odot}$ are likely to undergo an electron-capture supernova, while single stars in the same mass range would end as $\mathrm{ONeMg}$ white dwarfs. We suggest that the core collapse in an electron-capture supernova (and possibly in the case of relatively small iron cores) leads to a prompt or fast explosion rather than a very slow, delayed neutrino-driven explosion and that this naturally produces neutron stars with low-velocity kicks. This leads to a dichotomous distribution of neutron star kicks, as inferred previously, where neutron stars in relatively close binaries attain low kick velocities. We illustrate the consequences of such a dichotomous kick scenario using binary population synthesis simulations and discuss its implications. This scenario has also important consequences for the minimum initial mass of a massive star that becomes a neutron star. For single stars the critical mass may be as high as $10-12 M_{\odot}$, while for close binaries it may be as low as 6-8 $M_{\odot}$. These critical masses depend on the treatment of convection, the amount of convective overshooting, and the metallicity of the star, and will generally be lower for larger amounts of convective overshooting and lower metallicity.

Subject headings: binaries: close — globular clusters: general — stars: evolution — stars: neutron — supernovae: general $-\mathrm{X}$-rays: stars

On-line material: color figures
\end{abstract}

\section{INTRODUCTION}

For the last decade, it has generally been accepted that the high speeds inferred for isolated, normal radio pulsars imply that neutron stars (NSs) receive a large impulse, or "kick," at birth. Measured proper motions for $\simeq 100$ radio pulsars indicate typical kick speeds in excess of $100-200 \mathrm{~km} \mathrm{~s}^{-1}$ (Lyne \& Lorimer 1994; Hansen \& Phinney 1997; Cordes \& Chernoff 1998; Arzoumanian et al. 2002), although the functional form of the underlying speed distribution is poorly constrained. The physical mechanism that causes this kick is currently unknown, but is presumably the result of some asymmetry in the core collapse or subsequent supernova (SN) explosion (see, e.g., Pfahl et al. 2002c for discussion and references).

In apparent conflict with the high speeds of isolated radio pulsars, Pfahl et al. (2002c) identified a new class of high-mass X-ray binaries (HMXBs) in which the NSs must have been

\footnotetext{
${ }^{1}$ Department of Astrophysics, University of Oxford, Oxford OX1 3RH, UK; podsi@astro.ox.ac.uk.

2 Astronomical Institute, P.O. Box 80000, NL-3508 TA Utrecht, The Netherlands; n.langer@astro.uu.nl.

${ }^{3}$ Department of Physics and Center for Space Research, Massachusetts Institute of Technology, 77 Massachusetts Avenue, Cambridge, MA 021394307; sar@mit.edu.

4 Department of Astronomy and Astrophysics, University of Chicago, 5640 South Ellis Avenue, Chicago, IL 60637; and Theoretical Astrophysics Group, T-6, MS B227, Los Alamos National Laboratory, Los Alamos, NM 87545; alex@6.lanl.gov.

5 Chandra Fellow, Harvard-Smithsonian Center for Astrophysics, 60 Garden Street, Cambridge, MA 02138; epfahl@cfa.harvard.edu.
}

born with relatively low kick speeds. These HMXBs are distinguished by their long orbital periods $\left(P_{\text {orb }}>30\right.$ days $)$ and low eccentricities $(e \lesssim 0.2)$, with the prime example being $\mathrm{X} \mathrm{Per} / 4 \mathrm{U} 0352+309\left(P_{\text {orb }} \simeq 250\right.$ days, $\left.e \simeq 0.1\right)$. The orbits of these systems are sufficiently wide that tidal circularization is negligible, so that the observed eccentricities should reflect the conditions immediately after the SN explosion. Such low eccentricities essentially require that the NSs in these systems received low kick speeds of $\leqslant 50 \mathrm{~km} \mathrm{~s}^{-1}$.

Further observational evidence that at least some NSs receive low kicks at birth is provided by the fact that a large number of NSs are found in globular clusters; some massive globular clusters may contain more than $\sim 1000$ NSs (Pfahl et al. 2002b). Since the central escape velocity is generally $\$ 50 \mathrm{~km} \mathrm{~s}^{-1}$, essentially all of the NSs born in a globular cluster should escape from the cluster if they received a kick consistent with the kick distribution for single radio pulsars (for a detailed discussion of this so-called neutron star retention problem, see Pfahl et al. 2002b). If all of these NSs were originally born in massive binaries, this would alleviate the problem somewhat, since in this case the momentum imparted to the NS would be shared by the total mass of the system, leading to a correspondingly smaller space velocity of the postSN binary (Brandt \& Podsiadlowski 1995). However, while membership in a binary increases the number of NSs that can remain bound in the cluster significantly, the effect may not be large enough to explain the observed numbers unless clusters were much more massive at an earlier epoch than they are today (Drukier 1996; Pfahl et al. 2002b). 
To simultaneously account for the new class of HMXBs and the high speeds of radio pulsars, Pfahl et al. (2002c) suggested that NSs originating from progenitors that are single or members of wide binaries receive the conventional large kicks, while NSs born in close binaries receive small kicks (also see Katz 1975, 1983 and Hartman 1997 for earlier more ad hoc suggestions of a significant population of NSs with small natal kicks). Pfahl et al. (2002c) further argued that the proposed dichotomy between high and low kick speeds and its relation to the evolutionary history of the NS progenitor might be associated with the rotation rate of the collapsing core. However, as we show in this paper the core structure itself (e.g., its mass and composition) depends strongly on whether a star evolves in a close binary or in isolation. This can produce differences in the actual SN and may allow a prompt (or at least a fast) SN explosion mechanism to be successful in some cases; this in turn may lead to relatively low SN kicks.

The outline of this paper is as follows: In $\S 2$ we systematically discuss the differences in the core evolution for stars in close binaries and in single systems/wide binaries and discuss the implications for the core-collapse phase. In $\S 3$ we develop a general scheme for forming NSs in different types of systems and the expected differences in kick velocity, associating them with individual known systems or classes of systems. In $\S 4$ we discuss how this scheme can be tested observationally and suggest how further progress can be made on the theoretical side.

\section{BINARY EVOLUTION AND THE PRE-CORE- COLLAPSE CORE STRUCTURE OF MASSIVE STARS}

It is often naively assumed that the evolution of helium cores is the same irrespective of whether the core is surrounded by a hydrogen envelope or not, and that the final core structure will be similar in the two cases. However, binary evolution may affect the final pre-SN structure of a massive star in several fundamentally different ways: (1) the rate of rotation of the immediate pre-SN core; (2) the size of the helium core; (3) the occurrence of a second dredge-up phase at the beginning of the asymptotic giant branch (AGB); and (4) the $\mathrm{C} / \mathrm{O}$ ratio at the end of helium burning, which affects the strength of carbon burning and the final size of the iron core. These effects can dramatically change the condition of core collapse as first pointed out by Brown et al. (1999) and in particular Brown et al. (2001), who showed that the final iron cores of massive stars will be significantly smaller for stars that have lost their hydrogen-rich envelopes soon after the end of core hydrogen burning.

\subsection{Effects of Binary Evolution}

\subsubsection{The Role of Rotation}

While massive stars are generally rapid rotators on the main sequence, there are several efficient mechanisms by which they lose their angular momentum during their evolution. The final rotation rate of the core of a massive star depends on whether the star passed through a red supergiant (RSG) phase, during which the core will be effectively braked by the hydrodynamic and magnetic coupling to the slowly rotating envelope (Spruit \& Phinney 1998; Heger et al. 2004), and the wind mass loss during a subsequent Wolf-Rayet/helium star phase, which can be very efficient in extracting angular momentum from a helium star (Heger \& Woosley 2003) and slowing it down in the process. Pfahl et al. (2002c) argued that stars that lost their hydrogen-rich envelopes soon after the main-sequence phase (so-called early case B mass transfer ${ }^{6}$ ) might avoid this phase where the core is effectively braked and may still be rapidly rotating at the time of core collapse.

However, whether the star can avoid spin-down also depends on whether mass transfer is dynamically stable or unstable. In the case of stable case B Roche lobe overflow, Langer et al. (2003) showed that if the mass-losing star remains tidally locked to the orbit this provides a very efficient method of slowing down the rotation rate of the mass-losing star. On the other hand, in the case of unstable mass transfer, leading to a common envelope (CE) and spiral-in phase (Paczyński 1976), the spiral-in timescale is much shorter than any realistic synchronization timescale and tidal locking would not be expected. In this case, the core of the mass-losing star could still be rapidly rotating after the ejection of the CE. This implies that the scenario suggested by Pfahl et al. (2002c) probably requires late case B mass transfer (i.e., dynamically unstable mass transfer that leads to a CE phase before the core has been spun down significantly).

Another situation that may lead to a rapidly rotating core is the complete slow merger of two massive stars, in particular if it occurs after helium core burning, as in models for the progenitor of SN 1987A (Ivanova \& Podsiadlowski 2003) that predict a very rapidly rotating core for the immediate pre-SN star (also see Joss \& Becker 2003).

Finally, we note that if the exploding star is still accreting from a companion at the time of the SN one would also expected a rapidly rotating core (Langer et al. 2004).

As this discussion shows, binary interactions can significantly affect the final pre-SN rotation rate of the core of a massive star, although the details can be rather involved and are not completely understood. In addition, what is even less clear at the present time is how this affects the physics of the core collapse itself and in particular the magnitude of the kick imparted to the newborn NS.

\subsubsection{The Size of the Helium Core and the Second Dredge-Up}

The final size of the helium core depends strongly on the evolution of the hydrogen-rich envelope. During core helium burning, the helium core ordinarily grows substantially because of hydrogen burning in a shell (often the dominant nuclear burning source) that adds fresh helium to the core. On the other hand, in a binary a star may lose its hydrogen-rich envelope before helium burning (or early during the helium-burning phase). In this case, the helium core can no longer grow and may in fact shrink because of the strong stellar wind expected in the subsequent Wolf-Rayet phase (e.g., Woosley et al. 1995; Wellstein \& Langer 1999; Wellstein et al. 2001, hereafter WLB01; Pols \& Dewi 2002). Therefore, the final mass of the helium core will often be lower for stars in close binaries than in single systems/wide binaries (see Fig. 1).

Another factor that strongly affects the mass of the final helium core is the occurrence of a second dredge-up phase. When stars up to $\sim 11 M_{\odot}$ ascend the AGB, they generally experience a second dredge-up phase where the convective envelope penetrates deep into the hydrogen-exhausted core and dredges up a significant fraction of the helium core (Iben 1974). As a consequence, the size of the helium core can be

\footnotetext{
${ }^{6}$ It is common practice to distinguish among three evolutionary phases of the primary at the onset of mass transfer, following Kippenhahn \& Weigert (1967; see also Lauterborn 1970; Podsiadlowski et al. 1992). Case A evolution corresponds to core hydrogen burning; case B refers to the shell hydrogenburning phase, but prior to central helium ignition; and case $\mathrm{C}$ evolution begins after helium has been depleted in the core.
} 


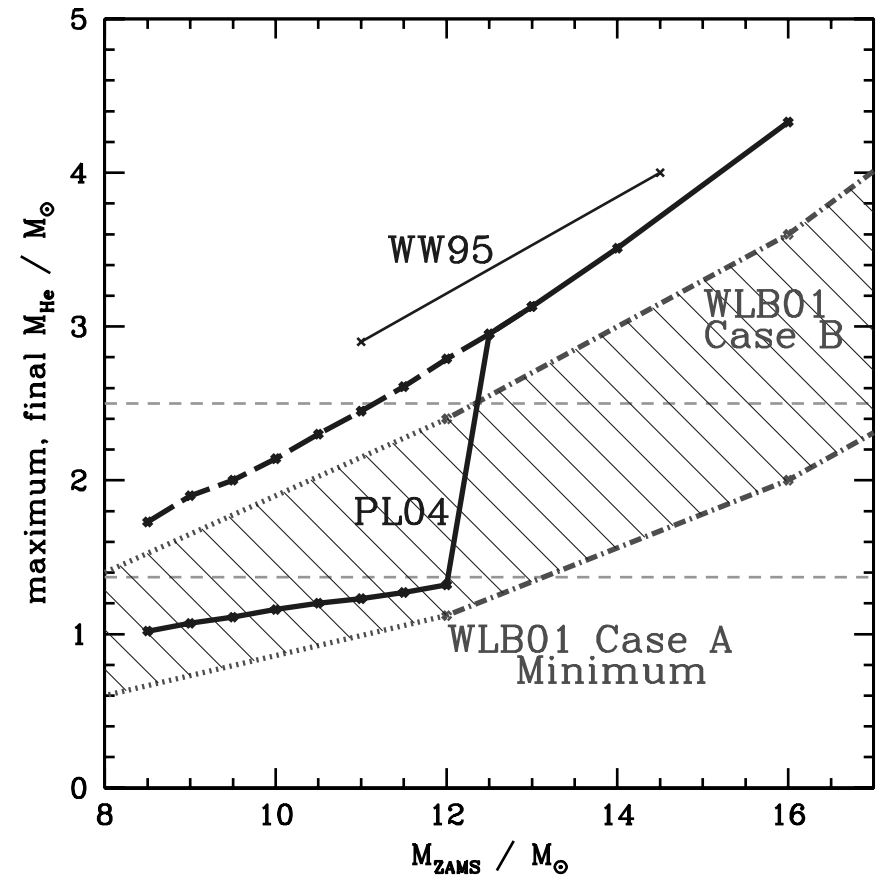

FIG. 1.-Final mass (thick solid line) and maximum mass (thick dashed line) of the helium core in single stars as a function of initial mass according to PL04. For comparison, the final helium core masses from the calculations of Woosley \& Weaver (1995, hereafter WW95) are indicated by a thin solid line. The final helium core masses of close binary models undergoing case B mass transfer from WLB01 are shown as the upper dot-dashed line, while those experiencing case A mass transfer (WLB01) may lie anywhere between the lower dot-dashed line and the case B line. The results from the binary calculations have been extrapolated for initial masses below $12 M_{\odot}$ (dotted part). Note that the PL04 and WLB01 models have been computed with the same assumptions for convective mixing, while the WW95 models assumed a higher semiconvective mixing efficiency. The light dashed horizontal lines give the range for the final helium core mass for which the star may experience an electron-capture SN. Note that the parameter range for which this may occur for a single star is very small. [See the electronic edition of the Journal for a color version of this figure.]

dramatically reduced (by up to $\sim 1.6 M_{\odot}$; see Fig. 1). However, the occurrence of the second dredge-up depends on the presence of a convective hydrogen-rich envelope. If the star loses its hydrogen-rich envelope before this phase, dredge-up does not occur. In this case, the final size of the helium core is larger for a star in a binary than its single counterpart (note that this is the opposite of what happens to their more massive counterparts). This is illustrated in Figure 1, which shows the final helium core mass as a function of initial main-sequence mass for single stars (thick solid curve; A. J. T. Poelarends \& N. Langer 2004, in preparation, hereafter PL04) and for stars in binaries that lose their envelopes either in case $\mathrm{A}$ or $\mathrm{B}$ mass transfer (based on the results of WLB01). The almost discontinuous change of the final helium mass around $12 M_{\odot}$ is a direct consequence of the fact that stars below this mass have experienced a second dredge-up phase, while stars above this mass do not or only dredge up a moderate amount of the helium core. Note that the final mass for these stars is less than the minimum mass for core collapse $\left(\sim 1.4 M_{\odot}\right)$. After the dredge-up phase, the helium core may grow again because of hydrogen shell burning, just as in a normal AGB star. Whether the core can reach the critical mass for core collapse depends on the timescale on which the star loses its envelope in a stellar wind or superwind. While this is somewhat uncertain, we estimate that for the models in Figure 1 single stars below $\sim 12 M_{\odot}$ will not reach the critical mass for core collapse and will end their evolution as ONeMg white dwarfs. There may be a small mass range around this boundary where single stars reach the condition for core collapse.

In contrast, the shaded region between the dot-dashed thick lines in Figure 1 indicates the expected range of the final helium core mass for stars that lose their envelopes by binary interactions (in this case, the final core mass depends on the evolutionary phase and the core mass at the time of mass transfer).

In the past, Nomoto (1984, 1987a) has argued that an electron-capture SN is the expected fate for stars with mainsequence masses in the range of $8-10 M_{\odot}$. In his calculations, these stars developed helium cores in the range of $M=2.0-$ $2.5 M_{\odot}^{7}$ and never developed an iron core; in this case, the collapse is triggered by the electron capture on ${ }^{24} \mathrm{Mg}$ and ${ }^{20} \mathrm{Ne}$ (Nomoto 1984, 1987a; for a recent discussion, see Wanajo et al. 2003). In Figure 1, the light dashed horizontal lines indicate approximately the range of helium core masses that can be expected to lead to an electron-capture SN (based on the results of Nomoto 1984, 1987a; also see Habets 1986). As Figure 1 shows, because of the second dredge-up the mass range for which single stars experience an electron-capture SN may be very narrow if nonexistent, while there is a large mass range for which it may occur for a star in a close binary. Indeed, a binary channel may be the only place where it can occur.

\subsubsection{The $\mathrm{C} / \mathrm{O}$ Ratio and the Strength of Carbon Burning}

Another more subtle effect is that the lack of a hydrogenburning shell leads to a lower $\mathrm{C} / \mathrm{O}$ ratio at the end of helium core burning, which affects the strength of subsequent carbon burning and the final size of the iron core. This effect was first pointed out and explained by Brown et al. (2001) and can be understood as follows.

In the late phase of helium core burning, the $\alpha$-capture reaction $\mathrm{C}^{12}+\alpha \rightarrow \mathrm{O}^{16}$, which destroys carbon, tends to dominate over the carbon-producing triple- $\alpha$ reaction (because of the different functional dependence on the number density of $\alpha$-particles). As a consequence, carbon is systematically being destroyed in this late core helium-burning phase (this switch occurs when the helium mass fraction falls below $Y=0.1-$ 0.2 ). In helium cores surrounded by a hydrogen-burning shell, both the hydrogen-exhausted core and the convective heliumburning core continue to increase during helium core burning. This leads to the continued injection of fresh helium into the helium-burning core and prolongs the phase in which carbon is preferentially destroyed. This eventually produces a smaller carbon abundance at the end of core helium burning than would be the case for a naked helium core, where the convective core does not grow. This has drastic consequences for the subsequent carbon-burning phase if helium or carbon-oxygen cores of similar sizes are compared. For high carbon abundances (as

\footnotetext{
${ }^{7}$ We note that in Nomoto's calculations, stars in the range of $8-10 M_{\odot}$ either did not experience a second dredge-up phase or did so only during carbon shell burning. As a consequence, most of his models in this mass range, unlike ours, experienced a core-collapse SN even in the case of single stars. We suspect that this difference can be attributed to the different opacities employed. The new OPAL opacities are significantly larger in the critical temperature range, which makes dredge-up more likely to occur earlier. We emphasize that this dredge-up behavior is qualitatively found in all other recent, detailed studies of stars in this mass range (Ritossa et al. 1996; GarcíaBerro et al. 1997; Iben et al. 1997; Eldridge \& Tout 2004). We note, however, that the range of initial masses that leads to helium cores in the range of 2.0$2.5 M_{\odot}$ depends on some of the uncertainties in the stellar modelling, in particular the treatment of convection and convective overshooting (for further discussion of the uncertainties, see $\S 2.3$ ).
} 
expected for naked helium cores), the phase of convective carbon shell burning lasts longer, typically leading to smaller carbon-exhausted cores. This in turn produces smaller iron cores with steeper density gradients outside the iron core (Fryer et al. 2002; Heger et al. 2002). Thus, for the same initial size of the helium or carbon core the higher carbon abundance in a star that lost its hydrogen-rich envelope before the central helium abundance dropped below $\sim 10 \%$ will result in a pre-SN structure that more easily produces a successful SN.

Brown et al. (2001) demonstrated that this dichotomy leads to much smaller iron cores for massive stars and that as a consequence even a $60 M_{\odot}$ main-sequence star may produce a NS rather than a black hole if it has lost its envelope before its helium core-burning phase. In contrast, the minimum mass for black hole formation for single stars may be as low as $20 M_{\odot}$. (Fryer 1999; Fryer \& Kalogera 2001).

In analogy to these results, it is reasonable to expect that there will be significant differences in the core properties even for massive stars with $M \lesssim 20 M_{\odot}$, possibly allowing for successful prompt (fast) SN explosions (see, e.g., Sumiyoshi et al. 2001 and $\S 2.2$ ).

\subsection{Prompt (Fast) Explosions and Supernova Kicks}

At present, neither the mechanism that produces a successful core-collapse SN nor the origin of SN kicks is properly understood (see Janka et al. 2003; Fryer 2003 for detailed recent reviews, and also Fryer \& Warren 2002, 2004). In one of the most popular explosion scenarios, it is the delayed heating by neutrinos that revives the outgoing SN shock several $100 \mathrm{~ms}$ after it stalled in the initial core bounce (i.e., after many dynamical timescales). In this scenario, the origin of the SN kick may be connected with asymmetries in this long phase where the explosion develops (e.g., because of the continued accretion onto the proto-NS or the strong convection in the neutrinoheated region; e.g., Janka et al. 2003). In contrast, in a prompt SN explosion the initial bounce drives a successful SN shock on the dynamical timescale of the proto-NS. The absence of a long phase where the explosion teeters at the brink of success could then be the cause for the absence of a large SN kick.

Such a scenario is supported by some of the most recent corecollapse simulations by Scheck et al. (2004; however, see also Fryer \& Warren 2004 for a very different view). In the simulations by Scheck et al. (2004), a large SN kick (up to and above $500 \mathrm{~km} \mathrm{~s}^{-1}$ ) is caused by asymmetries in the neutrino flux, which have their origin in low-order instabilities driven by the convective motion behind the stalled shock. An essential requirement in these simulations, which allows these convective instabilities to grow, is that the duration of the convective, stalled phase is longer than $\sim 500$ ms (i.e., many convective turnover timescales). Such slow explosions are expected for fairly large iron cores. In contrast, for small iron cores or in the case of electron-capture $\mathrm{SNe}$ the simulations by Scheck et al. (2004) suggest fast explosions where these convectively driven instabilities are unable to grow, leading to rather small kick velocities. $^{8}$

It seems quite attractive to relate the dichotomy in the SN kicks to the differences between a slow and a prompt (fast) SN explosion. Whether a SN explosion develops promptly or in a delayed manner depends mainly on the difference between the

\footnotetext{
${ }^{8}$ Note that, unlike the case of a prompt explosion, the fast explosions in the simulations by Scheck et al. (2004) occur on a timescale that is long compared to the dynamical timescale of the proto-NS.
}

mass of the $\mathrm{Fe}-\mathrm{Ni}$ core and the mass of the collapsing core (which in turn also depends on the initial entropy in the core), since this determines the amount of shock energy that is consumed in the nuclear dissociation of heavy elements (see Hillebrandt et al. 1984 for a detailed discussion and references; Sumiyoshi et al. 2001).

Electron-capture SNe provide a particularly promising scenario for a prompt (fast) explosion, since the whole core collapses to nuclear densities; this makes it much easier for the shock to eject the envelope, preventing the growth of the instabilities that lead to large kicks. As our previous discussion shows, electron-capture SNe may only (or mainly) occur in close binaries; in this case, NSs with low kicks may be (almost) exclusively produced in close binary systems (with orbital periods $\lesssim$ a few 100 days).

\subsection{The Minimum Mass for Core Collapse}

An important related issue is the question of the minimum initial mass $M_{\min }$ of a star that leads to a core-collapse SN in a single or binary system. It is commonly assumed that this minimum mass is around $8 M_{\odot}$, the minimum mass above which carbon ignites off-center (rather than explosively in the center) and an $\mathrm{ONeMg}$ core forms (Iben 1974). If the ONeMg core is able to grow to reach the Chandrasekhar mass, it will collapse in an electron-capture SN. However, if a star experiences mass transfer already on the main sequence, an initial star as massive as $\sim 16 M_{\odot}$ may end its evolution as a white dwarf rather than experience core collapse. ${ }^{9}$ On the other hand, as discussed above stars below $12 M_{\odot}$ may only experience core collapse if they have lost their envelopes by binary interactions after their main-sequence phase but before experiencing dredge-up on the AGB. This implies that the value of $M_{\min }$ may be as high as $12 M_{\odot}$ for single stars and as low as $8 M_{\odot}$ for relatively close binaries.

The exact value for $M_{\min }$ is quite sensitive to the treatment of convection and, in particular, the amount of convective overshooting and the metallicity of the star. The value of $M_{\min }=$ $12 M_{\odot}$ was obtained for models that used the Ledoux criterion for convection without convective overshooting. We estimate that using the Schwarzschild criterion would reduce $M_{\min }$ to $\sim 11 M_{\odot}$ if no convective overshooting is included, and to $\sim 10 M_{\odot}$ if the recent empirical calibration of convective overshooting (Pols et al. 1997; Schröder et al. 1997) is adopted (also see Ritossa et al. 1999; Eldridge \& Tout 2004). Similarly, the minimum mass for off-center carbon ignition may be as low as $\sim 6 M_{\odot}$ for models including convective overshooting (Z. Han 2002, unpublished).

Han et al. (1994) also found that for low-metallicity $(Z=$ $0.001)$ models without convective overshooting, the minimum mass for off-center carbon ignition was systematically lower $\left(\sim 6 M_{\odot}\right)$ than for solar metallicity $\left(\sim 8 M_{\odot}\right)$.

As these discussions illustrate, the initial mass range that leads to the formation of $\mathrm{ONeMg}$ white dwarfs, which also determines the minimum mass for stars that will experience core collapse, depends both on the details of binary interactions and on the stellar properties. Different treatments of convection are expected to lead to initial mass ranges ranging from $[6,9] M_{\odot}$ to $[9,12] M_{\odot}$. In addition, at low metallicity these

\footnotetext{
${ }^{9}$ This occurs when the system experiences an additional Roche lobe overflow phase after core helium exhaustion (case ABB mass transfer; WLB01; Podsiadlowski et al. 2003) during a helium-RSG phase; strong mass loss during the helium-RSG stage may lead to a similar outcome.
} 
ranges should be systematically shifted toward lower masses (by perhaps $2 M_{\odot}$ for $Z=0.001$; Han et al. 1994). Considering that this is an important parameter in galactic modelling, a thorough reexamination of this issue is urgently needed (PL04; Eldridge \& Tout 2004).

We emphasize that the exact values of this mass range do not affect the main arguments in this paper, since this only shifts the mass range in which an electron-capture SN can be expected but does not change the expected dichotomous behavior.

\section{A DICHOTOMOUS KICK SCHEME}

The scenario for NS kicks proposed herein has a significant impact on the theoretical production probabilities and distributions of orbital parameters of binary systems containing NSs. We illustrate this by means of a Monte Carlo binary population synthesis (BPS) calculation. Below we provide a brief description of the important elements of the code; further details may be found in Pfahl et al. (2002a, 2002b, 2002c, 2003). The BPS code follows a randomly generated sample of massive primordial binaries through the phase of mass transfer from the primary to the secondary. ${ }^{10}$ The initial primary and secondary masses, $M_{1 i}$ and $M_{2 i}$, are drawn from the respective distributions $p\left(M_{1 i}\right) \propto M_{1 i}^{-2.5}$ and $p\left(q_{i}\right)=1$, where $q_{i} \equiv M_{2 i} / M_{1 i}<1$ is the initial mass ratio. For simplicity we assume circular orbits, and the initial orbital separation $a_{i}$ is chosen from $p\left(a_{i}\right) \propto a_{i}^{-1}$.

Given some critical mass ratio, which we take to be $q_{c}=0.5$, the mass transfer from the primary to the secondary is assumed to be stable if $q_{i}>q_{c}$ and the envelope of the primary is mostly radiative when mass transfer begins (so-called cases $\mathrm{A}$, early $\mathrm{B}$, and early $\mathrm{C}$ ), and dynamically unstable if $q_{i}<q_{c}$ or the primary has a convective envelope (cases late $\mathrm{B}$ and late $\mathrm{C}$ ). In any case, we assume that the entire hydrogen-rich envelope of the primary is removed during mass transfer, leaving only the primary's hydrogen-depleted core.

For stable mass transfer, we suppose that the secondary accretes a fraction, $\beta=0.75$, of material donated by the primary, and that the remaining mass escapes the system with a specific angular momentum that is $\alpha=1.5$ times the orbital angular momentum per unit reduced mass. The value of $\alpha=$ 1.5 is characteristic of mass loss through the L2 point, but the choice of $\beta=0.75$ is fairly arbitrary, although it is convenient, since it gives $a_{f} / a_{i} \sim 1$, where $a_{f}$ is the final orbital separation. Dynamically unstable mass transfer results in a $\mathrm{CE}$ phase, wherein the secondary spirals into the envelope of the primary. The CE phase was treated in the same way as in Pfahl et al. (2002c). A fraction, $\eta_{\mathrm{CE}} \lesssim 1$, of the initial orbital energy is available to unbind the $\mathrm{CE}$ from the system. If insufficient energy is available, the two stars will merge. Otherwise, the envelope of the primary is dispersed, the secondary emerges near the zero-age main sequence (ZAMS) without having accreted any significant amount of mass, and the orbital separation is $\sim 100$ times smaller. A merger results in nearly every case where $q_{i}<q_{c}$ and the primary's envelope is radiative when mass transfer starts. A number of these evolutionary steps are illustrated in the top six panels of the schematic shown in Figure 2.

On exhausting its remaining nuclear fuel, the exposed core of the primary explodes as a Type Ib or Ic SN. We assume that the SN mass loss and natal NS kick are instantaneous, and that

\footnotetext{
${ }^{10}$ Here "primary" and "secondary" refer to the initially more and less massive star, respectively.
}

the orientations of the kicks are distributed isotropically. In light of the discussion above, the specific prescription adopted for NS kicks is as follows. Kick speeds are drawn from a Maxwellian distribution, with a mean that depends on the initial mass of the primary and its evolutionary state when it first fills its Roche lobe (see Fig. 2). If $8<M_{1 i}<14 M_{\odot}$ and mass transfer begins before helium ignition in the primary's core (cases A and B), the mean kick speed takes a small value of $30 \mathrm{~km} \mathrm{~s}^{-1}$, while in all other cases the mean is $300 \mathrm{~km} \mathrm{~s}^{-1}$. The range of masses and the actual mean kick speeds used here are reasonable choices, but are somewhat arbitrary and were chosen mainly for illustrative purposes.

The results of our BPS calculation are shown in Figure 3 in the $P_{\text {orb }}-M_{2}$ and $P_{\text {orb }}-e$ planes, where $M_{2}$ is the mass of the secondary star, including the mass it has accreted from the primary, and $e$ is the orbital eccentricity. Each small dot represents an incipient HMXB immediately after the SN explosion that produces the NS. The open circles represent systems that experienced the nominal natal NS kicks (see also left column of Fig. 2), while the crosses are systems where the primary lost its envelope early enough so that the natal kicks were much smaller, in accord with the dichotomous kick scenario proposed herein (see right column of Fig. 2).

The vertical strip in the $P_{\text {orb }}-M_{2}$ plane of Figure 3 results mostly from early case B systems in which the primaries had largely radiative envelopes and start mass transfer before core helium ignition. The resultant mass transfer is stable and the hydrogen-exhausted core evolves to a prompt (fast) SN explosion with a small natal kick. These systems generally acquire small eccentricities, especially for $P_{\text {orb }}$ in the range of 10-100 days. These are presumably the systems that evolve to become the newly identified class of HMXBs with relatively wide, loweccentricity orbits (e.g., X Per/4U 0352+30, $\gamma$ Cas/MX 0053+ 604, XTE J0543-568; Fig. 3, filled circles). For the systems of this type with still wider orbits, even the small natal kicks assumed here are sufficient to induce substantial eccentricities.

The "tail-shaped" region in the $P_{\text {orb }}-M_{2}$ plane of Figure 3 results from late case $\mathrm{B}$ systems commencing mass transfer when the primary had a large, convective envelope, but still before core helium ignition. These lead to $\mathrm{CE}$ events and result in systems with short orbital periods (i.e., $P_{\text {orb }}<10$ days; see, e.g., Fig. 2). Since such short-period systems would in any case be circularized by tidal friction, it may be difficult to distinguish these from their case $\mathrm{C}$ cousins in which the primaries were much more evolved at the start of mass transfer, and which received larger natal kicks. Systems in these latter categories (late case B and late case C) presumably include many of the famous HMXBs such as Cen X-3, LMC X-4, 4U 0900-40, and SMC X-1, with current-epoch (approximately) circular orbits and short orbital periods. The open circles in Figure 3 are those receiving a large kick, where the primary was highly evolved at the onset of mass loss (case C). These likely account for the class of eccentric HMXB systems with $P_{\text {orb }}$ in the range of 20-200 days, e.g., 4U 0115+63, GX 301-2, 4U 1145-619, and EXO 2030+375 (Bildsten et al. 1997; Fig. 3, filled triangles), and the three radio pulsars with massive companions in highly eccentric systems (Fig. 3, filled squares), PSR 1259-63 (Johnston et al. 1992), PSR J0045-7319 (Kaspi et al. 1994, 1996), and PSR J1740-3052 (Stairs et al. 2001).

Finally, we note that this dichotomous kick scenario helps the long-standing "retention problem" for NSs in globular clusters. In an earlier work (Pfahl et al. 2002b), we reported that our original dichotomous kick scenario yielded an enhancement factor of $\sim 4$ over the fraction of retained NSs 


\section{Dichotomous Kick Scenario}

\section{Large Natal Kick Small Natal Kick}

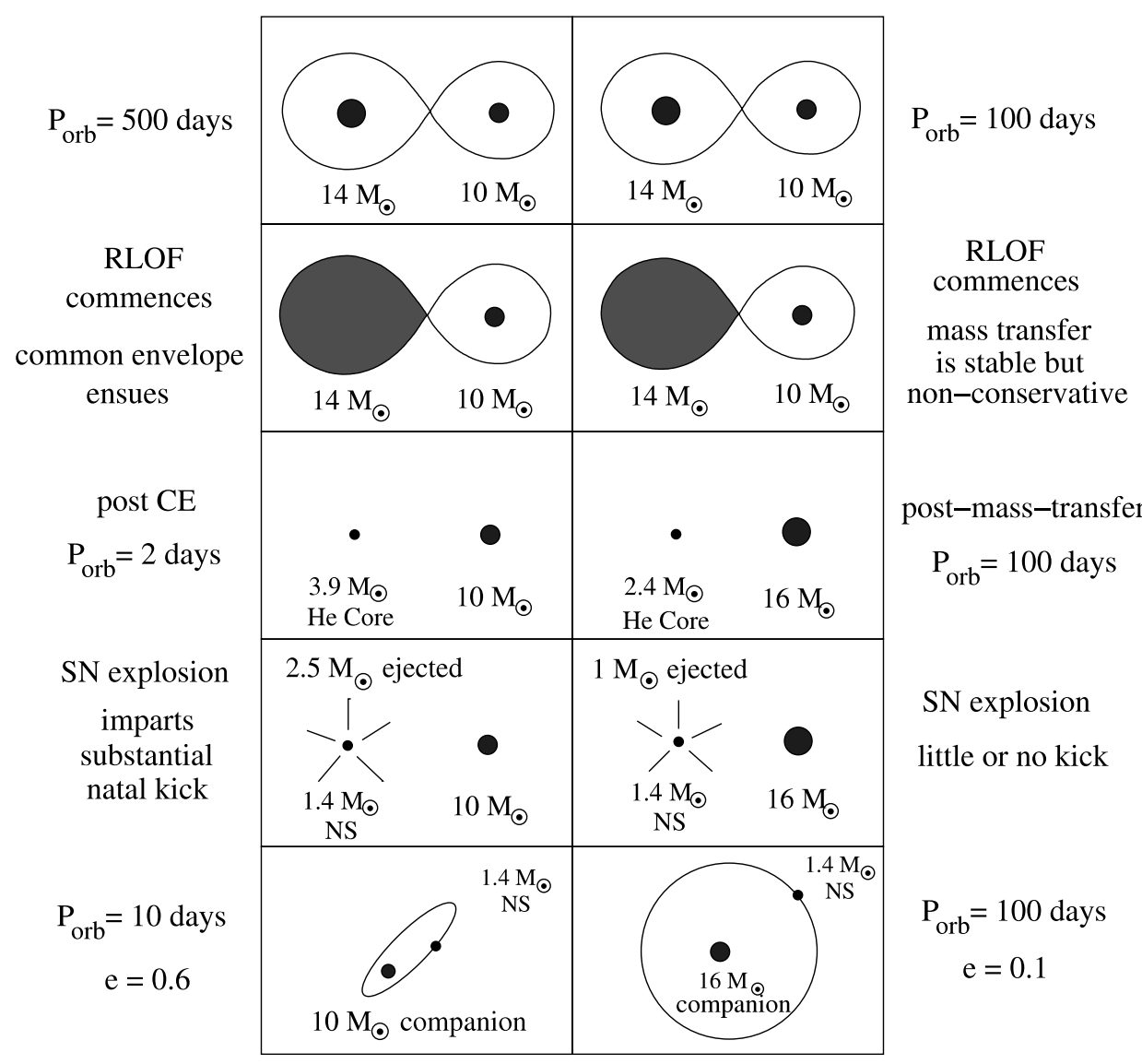

FIG. 2.-Illustrative binary evolution scenarios leading to the formation of HMXBs. In the left column, the binary is sufficiently wide at the onset of mass transfer

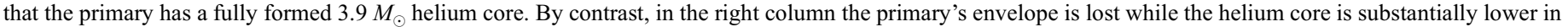

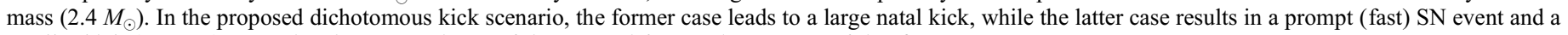
smaller kick (see text). [See the electronic edition of the Journal for a color version of this figure.]

without the appropriate subset of small kicks. We also pointed out (Pfahl et al. 2002b) that a dichotomous kick scenario would likely increase the theoretical formation rate of double-NS systems by approximately an order of magnitude. Both of these enhancements are expected to be carried over to this newer kick scenario.

\section{DISCUSSION AND FUTURE WORK}

As we have shown, the presence in a binary can dramatically affect the structure of the core of a massive star at the time of core collapse. Stars above $\sim 11 M_{\odot}$ are generally expected to have smaller iron cores if they lose their envelopes in a close binary. Stars in the range of $8-11 M_{\odot}$ may explode in an electron-capture SN if they are in a close binary, while single stars or stars in wide binaries will experience a second dredgeup phase and are more likely to end their evolution as $\mathrm{ONeMg}$ white dwarfs. We suggested that in the case of small iron cores or in the case of an electron-capture SN, the SN occurs through a prompt (fast) explosion rather than a delayed neutrino-driven explosion, and argued that this is more likely to produce NSs with low kick velocities.

While speculative at the moment, this scenario has important observational implications and suggests the need for further theoretical studies. These include a systematic exploration of the late evolution of the cores of stars around $10 M_{\odot}$, for stars evolved both in isolation and in a close binary; and of the dependence on metallicity and the amount of convective overshooting (e.g., PL04; Eldridge \& Tout 2004); and a reexamination of the core-collapse phase for electron-capture $\mathrm{SNe}$ and low-mass iron cores. One immediate prediction of this scenario is that NSs that formed from ONeMg or lowmass iron cores should produce NSs of systematically lower mass $\left(\sim 1.25 M_{\odot}\right.$ for a typical equation of state $\left.{ }^{11}\right)$. Wanajo et al. (2003) suggested that prompt explosions are the site for the $r$-process; if some of this $r$-processed matter is captured by the companion stars, this may produce detectable chemical anomalies in neutron star companions (e.g., X Per) similar to the case of the companion in Nova Scorpii (Israelian et al. 1999; Podsiadlowski, et al. 2002). Note also that in this case only small amounts of iron and other heavy elements are ejected in the SN. If all NSs in globular clusters were to form through such

11 The pulsar that formed last in the recently discovered double-pulsar binary PSR J0737-3039 (Burgay et al. 2003; Lyne et al. 2004) has a low mass of $\sim 1.25 M_{\odot}$, although at present it is not clear whether the formation of the second pulsar is consistent with a low-velocity kick. 


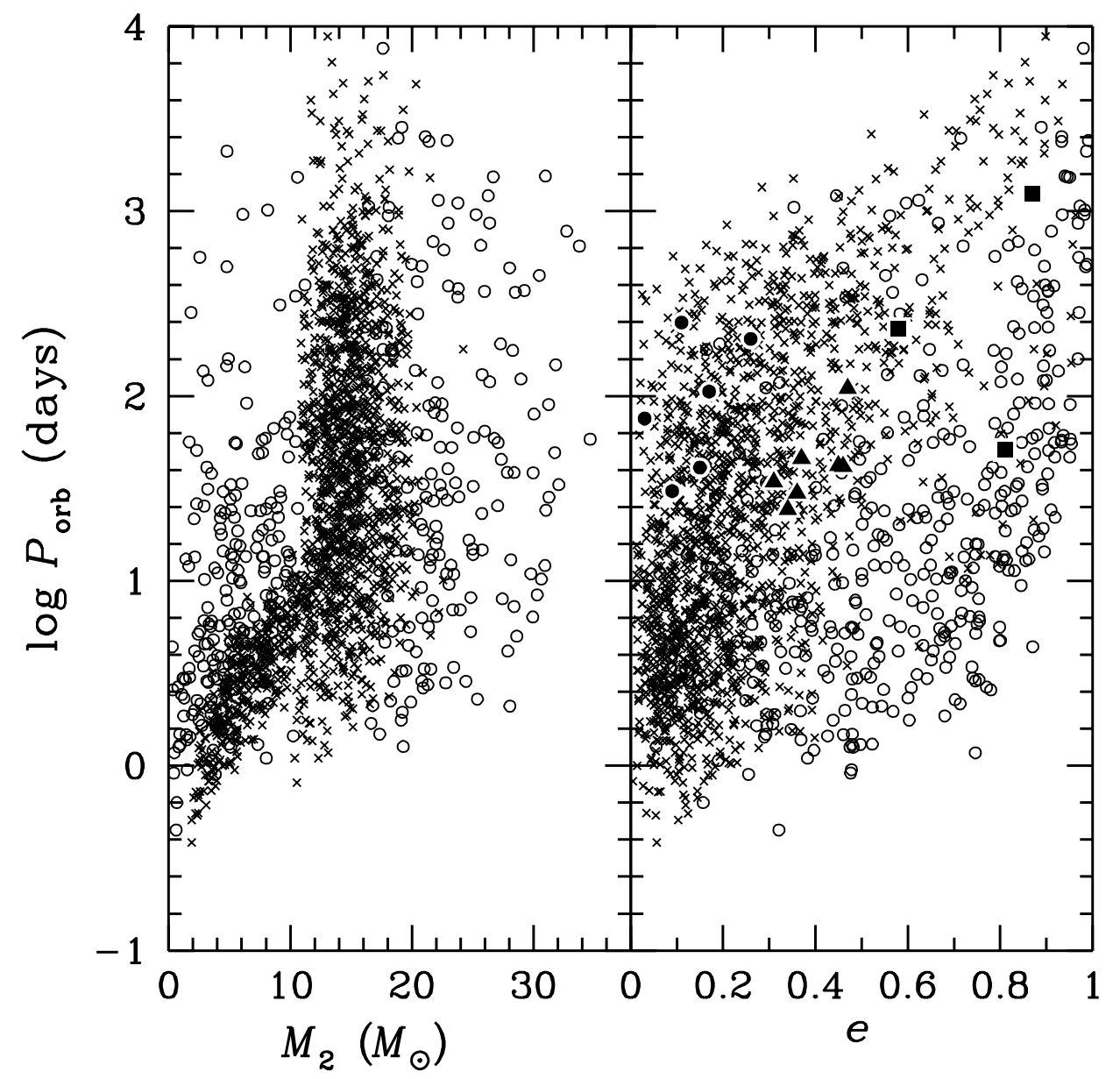

FIG. 3.-Results of a single HMXB population synthesis calculation that utilizes our proposed dichotomous kick scenario. Each open circle (cross) represents a system where the primary was (was not) highly evolved when it lost its hydrogen-rich envelope, and the exposed core evolved to collapse to form a NS with a subsequent large, conventional (small, unconventional) natal kick; see the text for details. Markers in the right panel indicate the observed wide, low-eccentricity HMXBs ( filled circles) and the well-known eccentric HMXBs ( filled triangles). The filled squares show the three radio pulsars with massive binary companions in eccentric orbits. We did not include observed systems with $P_{\text {orb }} \lesssim 10$ days, since their orbital parameters are likely to have been altered by tidal circularization effects. [See the electronic edition of the Journal for a color version of this figure.]

a channel, one would not expect significant chemical pollution of the cluster with heavy elements from SN ejecta (Price \& Podsiadlowski 1993).

Our scenario also has important implications for the minimum mass for neutron star formation; it suggests that only single stars more massive than $\sim 10-12 M_{\odot}$ become NSs, while in binaries the mass may be as low as $6-8 M_{\odot}$. The exact values will depend both on the amount of convective overshooting and the metallicity of the population; larger amounts of overshooting and lower metallicity are both expected to shift these critical masses to lower values. The detection of both young, single massive white dwarfs and NSs in binaries in open clusters with a turnoff mass around $10 M_{\odot}$ would provide direct evidence for such a dichotomy. Another promising method for observationally constraining the minimum mass for a core-collapse $\mathrm{SN}$ is through the detection of the progenitors of Type II-P SNe, stars that have not lost all of their hydrogenrich envelopes at the time of the SN explosion (see, e.g., Smartt et al. 2002).

Finally, it is worth mentioning another channel that is likely to produce an electron-capture SN and possibly a NS with a low kick if the basic picture described in this paper is correct: this involves the accretion-induced collapse (AIC) of a white dwarf to form a NS, either because an ONeMg white dwarf accreting from a companion star is pushed above the Chandraskekhar limiting mass (Nomoto 1987b; Nomoto \& Kondo 1991) or as the result of the merger of two CO white dwarfs, which is also likely to lead to the formation of a NS rather than a Type Ia SN (Nomoto \& Iben 1985). In principle, AIC could produce single low-velocity NSs with a rate as high as $3 \times 10^{-3} \mathrm{yr}^{-1}$ (Han 1998; Nelemans et al. 2001).

We thank H.-T. Janka and C. F. Fryer for very useful discussions. This work has been in part supported by the Netherlands Organization for Scientific Research (NWO) (A. J. T. P.), NASA ATP grant NAG5-12522 (S. R.), a Chandra Fellowship held at the Chandra X-Ray Center through grant PF2-30024 (E. P.), and the Department of Energy under contract W-7405-ENG-36 (A. H.). A. H. has also been supported in part by the Department of Energy under grant B341495 to the Center for Astrophysical Thermonuclear Flashes at the University of Chicago. 


\section{REFERENCES}

Arzoumanian, Z., Chernoff, D. F., \& Cordes, J. M. 2002, ApJ, 568, 289

Bildsten, L., et al. 1997, ApJS, 113, 367

Brandt, W. N., \& Podsiadlowski, Ph., 1995, MNRAS, 274, 461

Brown, G. E., Heger, A., Langer, N., Lee, C.-H., Wellstein, S., \& Bethe, H. 2001, NewA, 6, 457

Brown, G. E., Lee, C.-H., \& Bethe, H. A. 1999, NewA, 4, 313

Burgay, M., et al. 2003, Nature, 426, 531

Cordes, J. M., \& Chernoff, D. F. 1998, ApJ, 505, 315

Drukier, G. A. 1996, MNRAS, 280, 498

Eldridge, J., \& Tout, C. A. 2004, MNRAS, in press (astro-ph/0405408)

Fryer, C. L. 1999, ApJ, 522, 413 2003, in Stellar Collapse, ed. C. L. Fryer (Dordrecht: Kluwer)

Fryer, C. L., Heger, A., Langer, N., \& Wellstein, S. 2002, ApJ, 578, 335

Fryer, C. L., \& Kalogera, V. 2001, ApJ, 554, 548

Fryer, C. L., \& Warren, M. S. 2002, ApJ, 574, L65 2004, ApJ, 601, 391

García-Berro, E., Ritossa, C., \& Iben, I., Jr. 1997, ApJ, 485, 765

Habets, G. M. H. J. 1986, A\&A, 167, 61

Han, Z. 1998, MNRAS, 296, 1019

Han, Z., Podsiadlowski, Ph., \& Eggleton, P. P. 1994, MNRAS, 270, 121

Hansen, B. M. S., \& Phinney, E. S. 1997, MNRAS, 291, 569

Hartman, J. M. 1997, A\&A, 322, 127

Heger, A., \& Woosley, S. E. 2003, in AIP Conf. Proc. 662, Gamma-Ray Burst and Afterglow Astronomy (New York: AIP), 214

Heger, A., Woosley, S. E., Langer, N., \& Spruit, H. C. 2004, in IAU Symp. 215, Stellar Rotation, ed. A. Maeder \& P. Eenens (San Francisco: ASP), in press (astro-ph/0301374)

Heger, A., Woosley, S. E., Rauscher, T., Hoffman, R. D., \& Boyes, M. M. 2002, NewA Rev., 46, 463

Hillebrandt, W., Nomoto, K, \& Wolff, G. 1984, A\&A, 133, 175

Iben, I., Jr. 1974, ARA\&A, 12, 215

Iben, I., Jr., Ritossa, C., \& García-Berro, E. 1997, ApJ, 489, 772

Israelian, G., Rebolo, R., Basri, G., Casares, J., \& Martin, E. L. 1999, Nature, 401,142

Ivanova, N., \& Podsiadlowski, Ph. 2003, in From Twilight to Highlight: the Physics of Supernovae, ed. W. Hillebrandt \& B. Leibundgut (Berlin: Springer), 19

Janka, H.-T., Buras, R., Kifonidis, K., \& Rampp, M. 2003, in From Twilight to Highlight: the Physics of Supernovae, ed. W. Hillebrandt \& B. Leibundgut (Berlin: Springer), 39

Johnston, S., Manchester, R. N., Lyne, A. G., Bailes, M., Kaspi, V. M., Qiao, G., \& D’Amico, N. 1992, ApJ, 387, L37

Joss, P. C., \& Becker, J. A. 2003, in From Twilight to Highlight: the Physics of Supernovae, ed. W. Hillebrandt \& B. Leibundgut (Berlin: Springer), 104

Kaspi, V. M., Bailes, M., Manchester, R. N., Stappers, B. W., \& Bell, J. F. 1996, Nature, 381, 584

Kaspi, V. M., Johnston, S., Bell, J. F., Manchester, R. N., Bailes, M., Bessell, M., Lyne, A. G., \& D'Amico, N. 1994, ApJ, 423, L43

Katz, J. I. 1975, Nature, 253, 698

. 1983, A\&A, 128, L1
Kippenhahn, R., \& Weigert, A. 1967, Z. Astrophys., 65, 251

Langer, N., Wellstein, S., \& Petrovic J. 2003, in IAU Symp. 212, A Massive Star Odyssey: from Main Sequence to Supernova, ed. K. A. van der Hucht, A. Herrero, \& C. Esteban (San Francisco: ASP), 275

Langer, N., Yoon S.-C., Petrovic, J., \& Heger A. 2004, in IAU Symp. 215, Stellar Rotation, ed. A. Maeder \& P. Eenens (San Francisco: ASP), in press Lauterborn, D. 1970, A\&A, 7, 150

Lyne, A. G., \& Lorimer, D. R. 1994, Nature, 369, 127

Lyne, A. G., et al. 2004, Science, in press (astro-ph/0401086)

Nelemans, G., Yungelson, L. R., Portegies Zwart, S. F., \& Verbunt, F. 2001, A\&A, 365, 491

Nomoto, K. 1984, ApJ, 277, 791

-1987a, ApJ, 322, 206

1987b, in IAU Symp. 125, The Origin and Evolution of Neutron Stars,

ed. D. J. Helfand \& J.-H. Huang (Dordrecht: Kluwer), 281

Nomoto, K., \& Iben, I., Jr. 1985, ApJ, 297, 531

Nomoto, K., \& Kondo, Y. 1991, ApJ, 367, L19

Paczyński, B. 1976, in Structure and Evolution of Close Binary Systems, ed.

P. P. Eggleton, S. Mitton, \& J. Whelan (Dordrecht: Reidel), 75

Pfahl, E., Rappaport, S., \& Podsiadlowski, Ph. 2002a, ApJ, 571, L37 2002b, ApJ, 573, 283

2003, ApJ, 597, 1036

Pfahl, E., Rappaport, S., Podsiadlowski, Ph., \& Spruit, H. 2002c, ApJ, 574, 364

Podsiadlowski, Ph., Joss, P. C., \& Hsu, J. J. L. 1992, ApJ, 391, 246

Podsiadlowski, Ph., Nomoto, K., Maeda, K., Nakamura, T., Mazzali, P., \& Schmidt, B. 2002, ApJ, 567, 491

Podsiadlowski, Ph., Rappaport, S., \& Han, Z. 2003, MNRAS, 341, 385

Pols, O. R, \& Dewi, J. D. W. 2002, Publ. Astron. Soc. Australia, 19, 233

Pols, O. R., Tout C. A., Schröder K.-P., Eggleton, P. P., \& Manners, J., 1997, MNRAS, 289, 869

Price, N. M., \& Podsiadlowski, Ph., 1993, in ASP Conf. Ser. 48, The Globular Cluster-Galaxy Connection, ed. G. H. Smith \& J. P. Brodie (San Francisco: ASP), 721

Ritossa, C., García-Berro, E., \& Iben, I., Jr. 1996, ApJ, 460, 489 1999, ApJ, 515, 381

Scheck, L., Plewa, T., Janka, H.-T., Kifonidis, K., \& Müller, E. 2004, Phys. Rev. Lett., 92, 1103

Schröder, K.-P., Pols, O. R., \& Eggleton P. P., 1997, MNRAS, 285, 696

Smartt, S. J., Gilmore, G. F., Tout, C. A., \& Hodgin, S. T. 2002, ApJ, 565, 1089

Spruit, H. C., \& Phinney, E. S. 1998, Nature, 393, 139

Stairs, I. H., et al. 2001, MNRAS, 325, 979

Sumiyoshi, K., Terasawa, M., Matthews, G. J., Kajino, T., Yamada, S., \& Suzuki, H. 2001, ApJ, 562, 880

Wanajo, S., Tamamura, M., Itoh, N., Nomoto, K., Ishimaru, Y., Beers, T. C., \& Nozawa, S. 2003, ApJ, 593, 968

Wellstein, S., \& Langer, N. 1999, A\&A, 350, 148

Wellstein, S., Langer, N., \& Braun, H. 2001, A\&A, 369, 939 (WLB01)

Woosley, S. E., Langer, N., \& Weaver, T. A. 1995, ApJ, 448, 315

Woosley, S. E., \& Weaver, T. A. 1995, ApJS, 101, 181 (WW95) 